\title{
Effect of changes in an FFQ: comparing data from two national dietary survey instruments among 2-year-olds
}

\author{
A. L. Kristiansen ${ }^{1 *}$, I. T. L. Lillegaard ${ }^{1,2}$, B. Lande $^{3}$ and L. F. Andersen ${ }^{1}$ \\ ${ }^{1}$ Department of Nutrition, Institute of Basic Medical Sciences, University of Oslo, PO Box 1046 Blindern, \\ o316 Oslo, Norway \\ ${ }^{2}$ Norwegian Scientific Committee for Food Safety, Oslo, Norway \\ ${ }^{3}$ Division of Public Health, Norwegian Directorate of Health, Oslo, Norway \\ (Submitted 21 June 2011 - Final revision received 24 February 2012 - Accepted 24 February 2012 - First published online 4 May 2012)
}

\section{Abstract}

In the interpretation of dietary trends, it is important to consider the potential effect of modifications in the dietary assessment method. Therefore, our objective was to explore the comparability of data obtained at two time points by a semi-quantitative FFQ (SFFQ) which has had slight modifications over time. In the national dietary surveys among Norwegian 2-year-olds, diet was assessed by an SFFQ which underwent modifications between the 1999 survey and the 2007 survey. In the present study, fifty-nine families with a 2-year-old child participated by completing both the SFFQ in a crossover design within a month's time. With regard to the reported intake of energy and nutrients, the largest significant differences observed between the two questionnaires were for carbohydrates and added sugar. According to intake of food groups, significant differences were observed for five out of sixteen food groups. Spearman's correlation coefficients for energy, nutrients and food groups ranged from 0.43 (Ca) to 0.85 (soft drinks). Most Bland-Altman plots indicated broad limits of agreement. The differences between the two questionnaires can be explained by changes in the questionnaires, changes in the food composition databases used and random variation. Comparing differences between the questionnaires by maternal educational level, number of children and type of day care revealed minor differences. In conclusion, this study showed that at the group level there was reasonable comparability between the two questionnaires, except for carbohydrates, added sugar and some food groups. Moreover, there were moderate to high correlations for energy, nutrients and food groups.

\section{Key words: FFQ: Children: Dietary intake: National dietary surveys: Changes in FFQ}

Various methods have been used to assess dietary intake in infants and young children ${ }^{(1,2)}$. Dietary assessments are used to describe dietary intake, patterns and food sources, to estimate the adequacy of nutrient intake as well as to observe changes in dietary intake over time ${ }^{(1)}$. Assessment of dietary intake is also important when studying the relationship between diet and health $^{(3)}$. Moreover, nutrition monitoring information contributes to policy formulation ${ }^{(4)}$. In the interpretation of trends in dietary intake, there are a number of considerations to be taken into account; for example, the effect of slight modifications in the method over time, like improved visual aids, improvements in food composition databases and actual changes in the composition of foods ${ }^{(5)}$.

The first national dietary survey among Norwegian 2-yearolds was conducted in $1999^{(6)}$, whereas the second was conducted in $2007^{(7)}$. At both time points, diet was assessed with a semi-quantitative FFQ (SFFQ) (in 1999, the SFFQ-1999 and in 2007, the SFFQ-2007) completed by the mothers/parents. There were some differences between the SFFQ-1999 and the
SFFQ-2007. Therefore, the objective of the present paper was to explore the comparability of data obtained at the two time points due to slight modifications in the SFFQ over time.

\section{Study population and methods}

\section{Subjects and design}

A nationwide sample of 300 Norwegian 2-year-old children was established by the Norwegian Population Register. The sample included children born in Norway in the period from 18 April to 28 April 2006 of mothers born in Norway, Sweden or Denmark. If the child was a twin or a triplet, the mothers/parents were asked to include only the oldest. Informed consent was obtained from the mothers/parents. Those who gave a written refusal to participate were not contacted any further. The present study was conducted according to the guidelines laid down in the Declaration of Helsinki and all procedures involving human subjects were approved by the Regional

Abbreviations: E\%, percentage of energy; SFFQ, semi-quantitative FFQ.

*Corresponding author: A. L. Kristiansen, fax +47 228513 41, email a.l.kristiansen@medisin.uio.no 
Committees for Medical Research Ethics. Written informed consent was obtained from all subjects.

The mothers/parents were asked to complete two questionnaires, the SFFQ-1999 and the SFFQ-2007. The mothers/parents received an invitation letter and one of two SFFQ by mail about 2 weeks before the child turned 2 years of age. Close to the child's '2-years' birthday, all mothers/parents were contacted by telephone, both for clarifying possible questions and to motivate for participation in the study. The study had a crossover design where half of the sample received the SFFQ-1999 first and then about 2-3 weeks later they received the SFFQ2007. The second half received the SFFQ-2007 first and then about 2-3 weeks later they received the SFFQ-1999. Mothers/ parents were asked to complete the questionnaires as closely as possible to the child's '2-years' birthday. In accordance with the national surveys, they were asked to describe the habitual feeding practices and to keep the last $14 \mathrm{~d}$ in mind when filling in the questionnaire. To obtain data on the children's weight and height, mothers/parents were asked to bring the questionnaire to the regular 2-year check-up at the health clinic.

\section{The semi-quantitative FFQ}

The SFFQ-1999 was used as a template for the SFFQ-2007. Nevertheless, there were some differences between the SFFQ1999 and the SFFQ-2007. All modifications are shown in Table 1. As the validation study of the SFFQ-1999 against $7 \mathrm{~d}$ weighed records ${ }^{(8)}$ indicated overestimates of median intake of most nutrients and foods, the sequence of the frequency range and some frequency options were modified in the SFFQ-2007. In both questionnaires, the first frequency option was 'Never/less than once/month', however, the frequency range after this was different in the two questionnaires.

The two SFFQ included different number of questions regarding the use of food items (Table 1). In both questionnaires, these questions were grouped together according to the Norwegian meal pattern. As additions of jam and sugar etc. to porridge/ cereals were asked for in a separate question in the SFFQ2007, the SFFQ-2007 included one more question on food items compared to the SFFQ-1999. The difference between the questionnaires with regard to the number of food items asked about was mostly due to different assortment in groceries in 1999 and in 2007, as also due to change of some sub-questions. For example, the SFFQ-1999 included one sub-question about 'Semi whole grain and whole grain bread', while the SFFQ-2007 included 'Semi whole grain bread' and 'Whole grain bread' as two sub-questions. Furthermore, the SFFQ1999 included two sub-questions about 'Grilled/wiener sausage' and 'Minced meat sausage', while the SFFQ-2007 asked about: 'Grilled/wiener/minced meat sausage made from pork/ cattle', 'Grilled/wiener/minced meat sausage made from chicken/turkey' and 'Low-fat grilled/wiener/minced meat sausage'. Generally, the main questions on food items were more or less worded similarly in the two questionnaires.

To assist parents in reporting the amounts of food eaten, a photographic booklet was used. Minor modifications in the photographic booklet were made in 2007 (Table 1). The booklet included a colour photograph series with four different

Table 1. Modification in the semi-quantitative FFQ (SFFQ)-2007 compared to the SFFQ-1999, in the photographic booklet and the food composition databases used

\begin{tabular}{|c|c|c|}
\hline Modifications & SFFQ-1999 & SFFQ-2007 \\
\hline Frequency range & $\begin{array}{l}\text { Frequency range after 'never/less than once/month' } \\
\text { Often to seldom (e.g. times/d to times/week) }\end{array}$ & $\begin{array}{l}\text { Frequency range after 'never/less than once/month' } \\
\text { Seldom to often (e.g. times/week to times/d) }\end{array}$ \\
\hline \multirow[t]{12}{*}{ Frequency options } & Highest frequency option: & Highest frequency option: \\
\hline & Yoghurt: ' 4 or more times/d' & Yoghurt: '3 or more times/d' \\
\hline & Vegetables: ' 3 or more times/d' & Vegetables: '2 or more times/d' \\
\hline & Vitamin/mineral supplements: ' 3 or more times/d' & Vitamin/mineral supplements: '2 or more times/d' \\
\hline & Dinner dishes*: & Dinner dishes*: \\
\hline & 'Never/less than once/month' & 'Never/less than once/month' \\
\hline & '1 time/week' & '1 time/month' \\
\hline & ‘2 times/week’ & '2 times/month' \\
\hline & '3 times/week' & '3 times/month' \\
\hline & '4 times/week' & '1 times/week' \\
\hline & ' 5 or more times/week' & '2 times/week' \\
\hline & ‘1-3 times/month’ & '3 or more times/week' \\
\hline \multirow[t]{2}{*}{ Number of main questions } & Thirty-nine main questions & Forty-five main questions \\
\hline & $\begin{array}{l}\text { Thirteen main questions regarding } \\
\text { use of approximately } 140 \text { food items }\end{array}$ & $\begin{array}{l}\text { Fourteen main questions regarding } \\
\text { use of approximately } 160 \text { food items }\end{array}$ \\
\hline \multirow[t]{3}{*}{ Photographic booklet } & Sixteen photograph series & Seventeen photograph series \\
\hline & & $\begin{array}{l}\text { A new photograph series of sausages } \\
\text { was added }\end{array}$ \\
\hline & & $\begin{array}{l}\text { Rice photograph series replaced with } \\
\text { smaller portion sizes }\end{array}$ \\
\hline Food composition database used & Database used for nutrient calculations is IE-96 & Database used for nutrient calculations is $\mathrm{AE}-07$ \\
\hline & The energy content of foods does not & Dietary fibre contributes with $8 \mathrm{~kJ} / \mathrm{g}$ fibre \\
\hline & include energy from dietary fibre & Approximately 100 food item codes \\
\hline & & were the same as in SFFQ-1999 \\
\hline
\end{tabular}

*Dinner dishes refers to sixteen and nineteen different types of dinner foods like chicken, sausages, fish fingers, pancakes, pizza etc. in the SFFQ-1999 and SFFQ-2007, respectively. 
portion photographs ranging from small to large. In 2007, one photograph series of sausages was added. In addition, the photograph series of rice was replaced by a new series of rice with smaller portions than in 1999. When no photo was available for a food item, household units like slices, spoons etc. were used in both questionnaires. For most food items, the portion sizes were the same at both time points.

Both questionnaires also requested information like parental educational levels, maternal work situation, maternal marital status, number of children/parity, infant birth weight, gestational age, type of day care and how parents were informed on child nutrition. When the national dietary survey was conducted in $1999^{(6)}$, Statistics Norway provided information on child's sex and maternal age, whereas the SFFQ-2007 requested information on child's sex and maternal age, as well as maternal smoking habits. Due to this difference, the total number of main questions in the two questionnaires was different (Table 1).

\section{Nutrient calculations}

Daily intake of energy, nutrients and food groups was computed by using food databases in a software system (KBS) developed at the Institute of Basic Medical Sciences, Department of Nutrition, at the University of Oslo. The food databases are mainly based on various versions of the official Norwegian food composition table $\mathrm{e}^{(9-11)}$, and are continuously supplemented with data on new food items. The use of cod liver oil and vitamin/mineral supplements is included in the nutrient calculations. The database used for nutrient calculations of the SFFQ-1999 data was IE-96, while the database AE-07 was used for nutrient calculations of the SFFQ-2007 (Table 1). The effects of changing food composition database were investigated by analysing the SFFQ-1999 data with the same food composition database as the SFFQ-2007 (AE-07). In the database IE-96, the energy content of foods does not include energy from dietary fibre; however, in the database AE-07, dietary fibre contributes with $8 \mathrm{~kJ} / \mathrm{g}$ fibre. To be able to compare daily intake of energy in the two questionnaires, results from the SFFQ-2007 are presented as energy intake $(\mathrm{kJ})$ minus fibre intake $(\mathrm{g}) \times 8 \mathrm{~kJ}$.

\section{Statistical analyses}

All statistical analyses were performed by IBM ${ }^{\circledR}$ SPSS $^{\circledR}$ Statistics, version 18.0 (IBM Corporation). As the intake of nutrients and food groups was not normally distributed, non-parametric statistical methods were chosen. For both questionnaires, mean and median are presented for intake of energy, nutrients and food groups. Differences in intake were analysed using Wilcoxon's signed rank test (paired data). The relationship between the two SFFQ is presented by Spearman rank correlation coefficients. The visual agreement between measurements was analysed by the method proposed by Bland and Altman ${ }^{(12)}$, using a plot of the difference between two measurements against the average of the measurements. This type of plot shows the magnitude of disagreement, spot outliers and any trend within the variable. Furthermore, the analysis also assesses the agreement at individual level, defined as the limit of agreement ( $\pm 2 \mathrm{SD}$ of the mean). To inspect whether maternal educational level, number of children and type of day care at 2 years of age influenced our findings; mean differences in intake of energy, selected micronutrients and percentage of energy (E\%) from macronutrients were compared using the Kruskal-Wallis test and the MannWhitney $U$ test (unpaired data).

\section{Results}

Completed questionnaires (both SFFQ-1999 and SFFQ-2007) were received for fifty-nine of the 2 -year-olds (20\% response rate). Table 2 shows the characteristics of the participants. A total of thirty participants answered the SFFQ-1999 first, while twenty-nine participants answered the SFFQ-2007 first. No statistically significant differences with regard to characteristics of the participants were observed among those answering the SFFQ-2007 first compared to those answering the SFFQ1999 first (data not shown). Both questionnaires were generally completed within a month's time and by the same person (92\%), mostly by the mother.

\section{Nutrient intake}

Table 3 presents the mean percentage differences (and withinperson difference SD) for energy and nutrient intake between the SFFQ-1999 and the SFFQ-2007. Significant differences were found for absolute intake of carbohydrates, E\% from carbohydrates, E\% from protein, E\% from added sugar and absolute intake of added sugar. The largest difference was observed for added sugar. For energy and intake of other nutrients, there were no significant differences between the questionnaires. For energy and most nutrients, the mean percentage difference between the questionnaires was within $\pm 5 \%$. Most Bland-Altman plots for nutrients indicated broad limits of agreement, and had similar shape as the plot for energy (Fig. 1). The points on the Bland-Altman plots were scattered both above and below zero, indicating no systematic difference between the two questionnaires.

Table 2. Characteristics of the participants ( $n$ 59) (Number of participants and percentages)

\begin{tabular}{lll}
\hline Sex & $n$ & $\%$ \\
\hline Boys & 23 & 39 \\
$\quad$ Girls & 36 & 61 \\
Maternal education* & & \\
$\quad$ Low $†$ & 16 & 28 \\
$\quad$ High $\ddagger$ & 42 & 72 \\
Number of children§ & & \\
$\quad$ One child & 19 & 33 \\
$\quad$ Two children or more & 39 & 67 \\
Day care & & \\
$\quad$ Kindergarten & 30 & 51 \\
$\quad$ Kindergarten and parents/other & 17 & 29 \\
$\quad$ Parents/other & 12 & 20 \\
\hline
\end{tabular}

${ }^{*}$ Missing data $(n 1)$.

† Low education $=$ less than academy/college/university. $\ddagger$ High education $=$ academy/college/university or more. $\S$ Missing data (n 1). 
Table 3. Intake of energy, micro- and macronutrients by semi-quantitative FFQ (SFFQ)-2007 and SFFQ-1999, mean \% difference, within-person difference between the SFFQ and Spearman's correlation $(r)(n 59)$

(Means, medians and standard deviations)

\begin{tabular}{|c|c|c|c|c|c|c|c|c|c|}
\hline & \multicolumn{3}{|c|}{ SFFQ-2007 } & \multicolumn{3}{|c|}{ SFFQ-1999 } & \multirow{2}{*}{$\begin{array}{c}\text { Mean \% } \\
\text { difference } \\
(\%) \dagger\end{array}$} & \multirow{2}{*}{$\begin{array}{l}\text { Within-person } \\
\text { difference } \\
\text { (SD) }\end{array}$} & \multirow{2}{*}{$\begin{array}{c}\text { Spearman's } \\
\text { correlation } \\
(r)\end{array}$} \\
\hline & Mean & SD & Median & Mean & SD & Median & & & \\
\hline Energy (kJ) & $5397 \cdot 0$ & $1657 \cdot 9$ & $5258 \cdot 8$ & $5701 \cdot 9$ & $1526 \cdot 3$ & 5584.0 & $-5 \cdot 3$ & $1277 \cdot 9$ & 0.66 \\
\hline Fat $(\mathrm{g})$ & 47.5 & $16 \cdot 2$ & $46 \cdot 0$ & 48.4 & $14 \cdot 3$ & 48.9 & -1.9 & $12 \cdot 6$ & 0.63 \\
\hline Protein $(\mathrm{g})$ & $47 \cdot 3$ & $15 \cdot 9$ & $46 \cdot 4$ & $48 \cdot 0$ & $14 \cdot 6$ & $47 \cdot 7$ & -1.5 & $12 \cdot 7$ & 0.65 \\
\hline Carbohydrates (g) & $166 \cdot 9$ & $54 \cdot 1$ & $160 \cdot 3$ & $182 \cdot 2$ & 54.9 & $176 \cdot 7$ & $-8 \cdot 4^{\star}$ & 47.4 & 0.67 \\
\hline Added sugar (g) & $22 \cdot 4$ & $15 \cdot 2$ & 19.9 & 28.6 & $16 \cdot 2$ & $25 \cdot 8$ & $-21 \cdot 7^{\star \star}$ & $18 \cdot 0$ & 0.48 \\
\hline $\mathrm{Ca}(\mathrm{mg})$ & 732.5 & $306 \cdot 4$ & $662 \cdot 0$ & 739.6 & 270.5 & $701 \cdot 0$ & -1.0 & 278.5 & 0.43 \\
\hline $\mathrm{Fe}(\mathrm{mg})$ & $7 \cdot 0$ & $2 \cdot 7$ & 6.5 & $7 \cdot 4$ & 2.5 & 6.9 & $-5 \cdot 0$ & 2.5 & 0.54 \\
\hline Vitamin D $(\mu \mathrm{g})$ & $7 \cdot 4$ & $6 \cdot 1$ & $6 \cdot 2$ & $7 \cdot 8$ & $6 \cdot 1$ & 5.9 & -4.9 & 4.3 & 0.75 \\
\hline$\%$ Energy from fats & 32.4 & 5.5 & $32 \cdot 1$ & 31.5 & $5 \cdot 0$ & $31 \cdot 1$ & 2.9 & 4.9 & 0.54 \\
\hline$\%$ Energy from proteins & 14.9 & $2 \cdot 2$ & $15 \cdot 0$ & $14 \cdot 3$ & 1.9 & $14 \cdot 1$ & $4 \cdot 9^{* *}$ & 1.9 & 0.52 \\
\hline$\%$ Energy from carbohydrates & $52 \cdot 7$ & $5 \cdot 3$ & 52.5 & $54 \cdot 2$ & $5 \cdot 7$ & 53.8 & $-3 \cdot 0^{\star}$ & $5 \cdot 2$ & 0.54 \\
\hline$\%$ Energy from sugar & $7 \cdot 1$ & 3.8 & 6.5 & 8.6 & $4 \cdot 1$ & 8.2 & $-17 \cdot 4^{\star \star}$ & 3.9 & 0.52 \\
\hline
\end{tabular}

Values were significantly different: ${ }^{\star} P<0.05,{ }^{\star \star} P<0.01$ ( $P$ is based on Wilcoxon's signed rank test).

$\dagger$ Mean \% difference between the SFFQ $=($ mean difference (SFFQ-2007 - SFFQ-1999)/mean SFFQ-1999) $\times 100$.

Spearman correlation coefficients between pairwise measurements of the nutrient intake from the SFFQ-1999 and the SFFQ-2007 ranged from 0.43 for $\mathrm{Ca}$ to 0.75 for vitamin D. The median correlation coefficient for absolute nutrient intake was 0.64 (Table 3).

When the SFFQ-1999 data were analysed with the same food composition database as the SFFQ-2007 data (AE-07), the differences between the questionnaires were reduced for intake of energy and nutrients. Moreover, the differences in absolute intake and $\mathrm{E} \%$ from carbohydrates became non-significant. However, the significant difference in absolute intake and $\mathrm{E} \%$ from sugar persisted (data not shown).

\section{Food intake}

Comparisons between the questionnaires were made for sixteen food groups (Table 4). Significant differences in food intake were found for bread, vegetables, yoghurt, meat and oatmeal porridge. The mean difference according to food groups

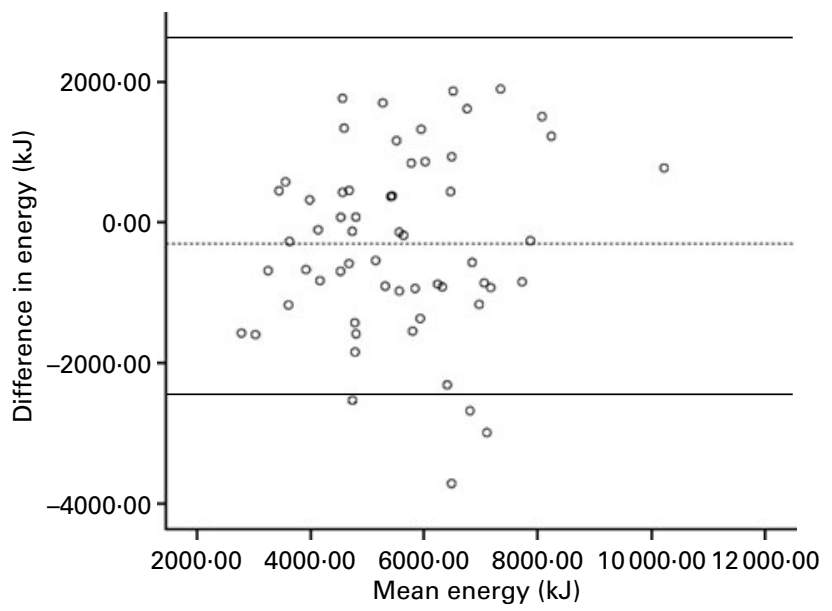

Fig. 1. Bland and Altman plot: energy intake (kJ) ( $n$ 59). Difference between energy intake estimated with semi-quantitative FFQ (SFFQ)-2007 and SFFQ-1999 plotted against the mean intake of energy from the two SFFQ. Dashed line represents mean difference, solid line represents mean \pm 2 SD. was within $\pm 20 \%$ for most food groups. Most Bland-Altman plots for food groups indicated broad limits of agreement, while some of the plots showed a tendency to increasing differences with increasing intake (data not shown).

Spearman correlation coefficients ranged from 0.55 for vegetables to 0.85 for soft drinks, while the median correlation coefficient for food intake was 0.68 (Table 4).

\section{Maternal education, number of children and type of day care}

Characteristics of the parents and children may affect the reporting of the dietary data. Therefore, observed differences between the questionnaires were analysed according to maternal educational level, number of children and type of day care. Data from children with mothers having low education ( $n$ 16) (less than academy/college/university) $v$. higher education ( $n$ 42) (academy/college/university or more) were compared. The mean differences in energy, selected micronutrients and $\mathrm{E} \%$ from macronutrients between the results from the two questionnaires were not significantly different between the two educational groups. No significant differences were observed when data from those with one child ( $n$ 19) were compared with data from those with two or more children ( $n$ 39). Data from the group of children who stayed at home with the parents/other care persons ( $n$ 12), the group of children with both day care by kindergarten and day care by parents/other care persons ( $n$ 17) and the group of children with day care only by kindergarten ( $n 30)$ were also compared. The type of day care seemed only to influence the reporting of the dietary data in a minor way. However, significant differences between groups were found for vitamin $\mathrm{D}(P=0.040)$ and $\mathrm{E} \%$ from fat $(P=0.046)$ (data not shown).

\section{Discussion}

The present study showed reasonable comparability between the two SFFQ used in the national dietary surveys in 1999 and in 2007. However, at the group level, substantial and significant 
Table 4. Intake of selected food groups by semi-quantitative FFQ (SFFQ)-2007 and SFFQ-1999, mean \% difference, within-person difference between the SFFQ and Spearman's correlation $(r)(n 59)$

(Means, medians and standard deviations)

\begin{tabular}{|c|c|c|c|c|c|c|c|c|c|}
\hline \multirow[b]{2}{*}{ Intake $(\mathrm{g} / \mathrm{d})$} & \multicolumn{3}{|c|}{ SFFQ-2007 } & \multicolumn{3}{|c|}{ SFFQ-1999 } & \multirow{2}{*}{$\begin{array}{c}\text { Mean \% } \\
\text { difference (\%) }\end{array}$} & \multirow{2}{*}{$\begin{array}{l}\text { Within-person } \\
\text { difference (SD) }\end{array}$} & \multirow{2}{*}{$\begin{array}{l}\text { Spearman's } \\
\text { correlation }(r)\end{array}$} \\
\hline & Mean & SD & Median & Mean & SD & Median & & & \\
\hline Water & 291.6 & $182 \cdot 0$ & $240 \cdot 0$ & 275.6 & 179.1 & $240 \cdot 0$ & $5 \cdot 8$ & 124.0 & 0.71 \\
\hline Milk (to drink) & 262.5 & 225.9 & $240 \cdot 0$ & 228.4 & 194.5 & 180.0 & 14.9 & 186.0 & 0.56 \\
\hline Fruit & $160 \cdot 8$ & $107 \cdot 6$ & $134 \cdot 3$ & 174.0 & $147 \cdot 2$ & $125 \cdot 7$ & -7.6 & 129.4 & 0.64 \\
\hline Bread & $128 \cdot 4$ & 60.4 & 126.0 & 111.2 & 44.9 & $108 \cdot 7$ & $15 \cdot 5^{\star \star}$ & 40.5 & 0.80 \\
\hline Fruit juices & 80.7 & 113.5 & $42 \cdot 6$ & $100 \cdot 7$ & $126 \cdot 1$ & 60.0 & -19.9 & $96 \cdot 8$ & 0.75 \\
\hline Yoghurt & $76 \cdot 1$ & 57.4 & $66 \cdot 7$ & 108.8 & 103.6 & 88.8 & $-30 \cdot 1^{\star}$ & 93.7 & 0.63 \\
\hline Squash & $50 \cdot 9$ & 79.1 & 17.4 & $65 \cdot 3$ & 132.9 & $17 \cdot 4$ & $-22 \cdot 1$ & $115 \cdot 0$ & 0.67 \\
\hline Vegetables & $49 \cdot 7$ & $36 \cdot 0$ & $45 \cdot 3$ & $42 \cdot 0$ & 42.4 & $32 \cdot 2$ & $18 \cdot 3^{*}$ & $41 \cdot 7$ & 0.55 \\
\hline Meat & $44 \cdot 1$ & $27 \cdot 7$ & $39 \cdot 3$ & $54 \cdot 2$ & 44.0 & 42.4 & $-18 \cdot 6^{\star}$ & 33.2 & 0.62 \\
\hline Potatoes & $29 \cdot 6$ & 23.7 & $20 \cdot 8$ & 29.9 & $21 \cdot 1$ & $22 \cdot 0$ & -1.0 & $17 \cdot 0$ & 0.74 \\
\hline Fish & $21 \cdot 7$ & $14 \cdot 4$ & 21.9 & $21 \cdot 1$ & $17 \cdot 2$ & $17 \cdot 2$ & $2 \cdot 8$ & $14 \cdot 1$ & 0.65 \\
\hline Oatmeal porridge & 18.9 & 35.4 & 0.0 & $25 \cdot 5$ & $47 . \overline{4}$ & 0.0 & $-25 \cdot 9^{*}$ & $26 \cdot 2$ & 0.72 \\
\hline Soft drinks & $16 \cdot 7$ & $27 \cdot 8$ & 0.0 & $15 \cdot 2$ & $19 \cdot 7$ & 0.0 & 9.9 & $17 \cdot 6$ & 0.85 \\
\hline Butter (on bread) & $13 \cdot 8$ & 8.4 & $15 \cdot 0$ & 13.4 & 8.0 & 13.5 & 3.0 & $6 \cdot 3$ & 0.68 \\
\hline Cakes & $12 \cdot 0$ & $11 \cdot 1$ & $10 \cdot 3$ & $10 \cdot 8$ & 8.4 & 9.4 & 11.1 & $8 \cdot 3$ & 0.62 \\
\hline Cod liver oil & 1.8 & 2.4 & 0.0 & 1.9 & $2 \cdot 4$ & 0.0 & $-5 \cdot 3$ & 1.7 & 0.75 \\
\hline
\end{tabular}

Values were significantly different: ${ }^{*} P<0.05,{ }^{*} P<0.01$ ( $P$ is based on Wilcoxon's signed rank test).

$\dagger$ Mean \% difference between the SFFQ $=$ (mean difference (SFFQ-2007 - SFFQ-1999)/mean SFFQ-1999) - 100.

differences were observed for carbohydrates and added sugar, with lower intake reported with the SFFQ-2007 compared to the SFFQ-1999. For food intake, significant differences were reported for five out of sixteen food groups: lower intake for yoghurt, meat and oatmeal porridge and higher for bread and vegetables with the SFFQ-2007 compared to the SFFQ-1999. There were moderate to high correlations, ranging from 0.43 to $0 \cdot 85$, for energy, nutrients and food groups.

The mean intake of energy was not significantly different in the SFFQ-2007 compared to the SFFQ-1999. Significant differences in $\mathrm{E} \%$ from proteins between the questionnaires were observed; however, this was probably due to the difference in reported intake of energy between the two questionnaires, even if this difference was not significant.

For intake of nutrients, the largest difference was seen for added sugar, both absolute intake and $\mathrm{E} \%$. A plausible explanation for this difference was the reduction in content of added sugar in flavoured yoghurt in the period from 1999 to 2007, as flavoured yoghurt was the main source of added sugar in both questionnaires. Thus, the content of added sugar in most flavoured yoghurt was reduced with about $3 \mathrm{~g}$ sugar/ $100 \mathrm{~g}$ yoghurt. Both the absolute intake and the $\mathrm{E} \%$ from carbohydrates were reported significantly lower in the SFFQ2007 compared to the SFFQ-1999. A likely explanation could be the significant difference in the intake of yoghurt, as yoghurt was an important source of carbohydrates in both questionnaires, contributing to 6 and $9 \%$ of the carbohydrate intake in the SFFQ-2007 and the SFFQ-1999, respectively. The difference in the intake of fruit could also contribute to explaining this difference. Fruit was the second most important contributor to the intake of carbohydrates in both questionnaires and a higher intake of fruit was reported with the SFFQ-1999 compared to the SFFQ-2007, although the difference was not significant. Even though bread was the main source of carbohydrates in both questionnaires, contributing to about $30 \%$ of the carbohydrate intake in both questionnaires, the difference in intake of carbohydrates could not be explained by the difference in intake of bread, as a significantly higher intake of bread was reported in the SFFQ-2007 compared to the SFFQ-1999. As added sugar is included in the total intake of carbohydrates, the lower intake of added sugar reported with the SFFQ-2007 compared to the SFFQ1999 could also explain the difference in carbohydrate intake.

In a reproducibility study of an FFQ among 124 Flemish preschoolers aged 2.5-6.5 years, Huybrechts et al. ${ }^{(13)}$ reported mean differences within a range of $\pm 10 \%$ for most of the evaluated food groups. However, none of the differences was significant. In the present study, we observed a mean difference according to food groups within a range of $\pm 20 \%$ for most food groups. Moreover, significant differences were observed for five out of sixteen food groups. Intake of bread and vegetables was reported significantly higher in the SFFQ-2007 compared to the SFFQ-1999. A reasonable explanation for this is the extended number of these foods in the SFFQ-2007. The SFFQ2007 asked about 'Semi whole grain bread' and 'Whole grain bread' in two sub-questions, while this was combined in one sub-question in the SFFQ-1999. With regard to intake of vegetables, there was addition in the SFFQ-2007 of six new subquestions on specific types of vegetables: tomato, cucumber, paprika, spinach, sweet corn and peas. A higher reported intake with extended number of food items is consistent with the work of Krebs-Smith et al. ${ }^{(14)}$, who observed how estimates of fruit and vegetable intake, from FFQ, were affected by the number of foods included.

There could be several reasons for the significantly higher intake of yoghurt reported with the SFFQ-1999 compared to the SFFQ-2007. Both questionnaires included six sub-questions on different types of yoghurt. The main difference between the 
sub-questions in the two questionnaires was the inclusion of a sub-question on non-specific fruit-flavoured yoghurt ('other fruit-flavoured yoghurt') in the SFFQ-1999. Inclusion of this sub-question might have caused an overestimation of intake of fruit-flavoured yoghurt in the SFFQ-1999. On the contrary, by not including this sub-question, the SFFQ-2007 may not be capable of capturing the intake of all fruit-flavoured yoghurt. The difference in the highest frequency option (" 4 or more times/d' in the SFFQ-1999 compared to ' 3 or more times/d' in the SFFQ-2007) (Table 1) could probably not explain the difference in intake of yoghurt, as the frequency option ' 4 or more times/d' in the SFFQ-1999 was only used in one sub-question by two participants.

Intake of meat was reported significantly higher in the SFFQ1999 compared to the SFFQ-2007. A slightly higher intake with regard to all meat dishes was observed in the SFFQ-1999 compared to the SFFQ-2007 and a reasonable explanation is the difference in the frequency alternatives according to dinner dishes (Table 1). The reported difference in intake of meat could also be explained by a higher reported intake of sausages in the SFFQ-1999 compared to the SFFQ-2007. In turn, this could be explained by the difference in portion size, as the SFFQ-1999 had larger portion sizes of sausages compared to the SFFQ-2007. Moreover, in the SFFQ-1999, household units were used to report the amount of sausage, while a photograph sequence was included in the photographic booklet used in the SFFQ-2007.

Significantly lower intake of oatmeal porridge was reported with the SFFQ-2007 compared to the SFFQ-1999. As there were no changes for frequency options or portion sizes between the two questionnaires, this might reflect the variation in intake between the reference periods, even though this should be minimised by the crossover design. In addition, a comparison including only one food item may be more prone to variation between the reference periods than comparisons including several food items.

According to intake of energy, nutrients and food groups, the correlations between the two questionnaires observed in our study were moderate to high $(0.43-0.85)$. The same has been observed in the reproducibility studies by Huybrechts et $a l^{(13)}$ and Metcalf et $a l^{(15)}$. Somewhat lower correlations were observed in a reproducibility study of an FFQ among forty-four preschool children with high migrant status in Switzerland ${ }^{(16)}$.

In most Bland-Altman plots, the limits of agreement were large for all nutrients and food groups. This indicates that the agreement at the individual level was of considerable variability.

In summary, the differences between the two questionnaires can be explained by changes in the questionnaires, changes in the food composition databases used and random variation.

\section{Strengths and weaknesses of the study}

An important strength of the present study is the crossover design. As others have found a tendency of higher reported intake in the first questionnaire compared to the second questionnaire $^{(13,15)}$ and because of the crossover design, the order of the questionnaires did not confound our findings. In addition, as the time period between answering the question- naires was within a month's time, we assumed this time to be sufficient so that the participants would not remember their answers from the first questionnaire when filling in the second questionnaire. We also assumed that the dietary habits of the 2-year-olds would not change considerably during this short period of time, even if these changes would be minimised by the crossover design.

The accuracy of the results obtained will depend on the participants' ability to precisely recall their child's diet at the two time points. Although a majority of the results reported in the in-home setting involved well-educated parents, Livingstone \& Robson ${ }^{(17)}$ conclude that parents can be reliable reporters of their children's diet at home, but not reliable reporters according to intake out-of-home. Maternal education, number of children or type of day care did not seem to influence the reporting of the dietary data in any major way. However, significant differences between groups according to day care situation were found for vitamin D and $\mathrm{E} \%$ from fat. It is though important to confirm these results in larger studies, as some of the subgroups in our study were quite small.

In the last few decades, there has been a tendency towards a decrease in the response rates of most health studies ${ }^{(18)}$. This study was no exception, where only $20 \%$ of the originally drawn sample was willing to participate. Due to the low participation rate, the participants were older and better educated compared to the mothers in the two national dietary surveys, and the sample may have reported more accurately than a random sample of families with a 2 -year-old child.

In conclusion, this study showed that at the group level there was reasonable comparability between the two questionnaires, except for carbohydrates, added sugar and some food groups. Moreover, there were moderate to high correlations for energy, nutrients and food groups.

\section{Acknowledgements}

The present study was generously supported by EXTRA funds from the Norwegian Foundation for Health and Rehabilitation. The authors are grateful to all children and their parents who participated in this study, and wish to thank Elin Sørra for help with collecting the dietary data. A. L. K. carried out the data analyses and wrote the manuscript. I. T. L. L. was responsible for the design and the field work. I. T. L. L., B. L. and L. F. A. assisted and provided advice during all the stages of the work. All authors contributed in the discussion and interpretation of the results, and in the drafting and editing of the manuscript. The authors declare no conflict of interest.

\section{References}

1. Burrows TL, Martin RJ \& Collins CE (2010) A systematic review of the validity of dietary assessment methods in children when compared with the method of doubly labeled water. J Am Diet Assoc 110, 1501-1510.

2. Ortiz-Andrellucchi A, Henríquez-Sánchez P, Sánchez-Villegas A, et al. (2009) Dietary assessment methods for micronutrient intake in infants, children and adolescents: a systematic review. BrJ Nutr 102, Suppl. 1, S87-S117.

3. Kant AK (2010) Dietary patterns: biomarkers and chronic disease risk. Appl Physiol Nutr Metab 35, 199-206. 
4. McGinnis JM, Harrell JA \& Meyers LD (1990) Nutrition monitoring: interface of science and policy. J Nutr 120, Suppl. 11, $1437-1439$

5. Briefel RR \& Johnson CL (2004) Secular trends in dietary intake in the United States. Annu Rev Nutr 24, 401-431.

6. Lande B \& Andersen LF (2009) Småbarnskost - Dietary Habits among 2-Year-old Children in Norway (in Norwegian). Report no. IS-1299. Oslo: Norwegian Directorate of Health.

7. Kristiansen AL, Lande B \& Andersen LF (2009) Småbarnskost 2007 - Dietary Habits among 2-Year-old Children in Norway (in Norwegian). Report No. IS-1731. Oslo: Norwegian Directorate of Health.

8. Andersen LF, Lande B, Trygg K, et al. (2004) Validation of a semi-quantitative food-frequency questionnaire used among 2-year-old Norwegian children. Public Health Nutr 7, 757-764.

9. Rimestad AH, Løken EB \& Nordbotten A (2000) The Norwegian food composition table and calculation system used at the Institute for Nutrition Research. Nor J Epidemiol 10, $107-110$.

10. The Norwegian Food Safety Authority, The Norwegian Directorate of Health \& University of Oslo (2006) The Norwegian Food Composition Table 2006. http://www. matportalen.no/matvaretabellen
11. National Nutritional Council \& The Food Control Authority (1995) The Norwegian Food Composition Table 1995 (in Norwegian). Oslo: Universitetsforlaget.

12. Bland JM \& Altman DG (1986) Statistical methods for assessing agreement between two methods of clinical measurement. Lancet i, 307-310.

13. Huybrechts I, De Backer G, De Bacquer D, et al. (2009) Relative validity and reproducibility of a food-frequency questionnaire for estimating food intakes among Flemish preschoolers. Int J Environ Res Public Health 6, 382-399.

14. Krebs-Smith, Heimdinger J, Subar AF, et al. (1995) Using food frequency questionnaires to estimate fruit and vegetable intake: association between the number of questions and total intakes. J Nutr Educ 27, 80-85.

15. Metcalf PA, Scragg RK, Sharpe S, et al. (2003) Short-term repeatability of a food frequency questionnaire in New Zealand children aged 1-14y. Eur J Clin Nutr 57, 1498-1503.

16. Ebenegger V, Marques-Vidal P, Barral J, et al. (2010) Eating habits of preschool children with high migrant status in Switzerland according to a new food frequency questionnaire. Nutr Res 30, 104-109.

17. Livingstone MB \& Robson PJ (2000) Measurement of dietary intake in children. Proc Nutr Soc 59, 279-293.

18. Antonsen S (2005) The motivation to participate in health studies (in Norwegian). J Nutr Educ 15, 99-109. 